\title{
Successful minimally invasive surgery for primary hyperparathyroidism: influence of preoperative imaging and intraoperative parathyroid hormone levels
}

\author{
Gaëtan-Romain Joliat ${ }^{1}$ • Nicolas Demartines ${ }^{1}$ - Luc Portmann ${ }^{2} \cdot$ Ariane Boubaker $^{3}$. \\ Maurice Matter ${ }^{1}$
}

Received: 15 April 2015 / Accepted: 8 November 2015 /Published online: 21 November 2015

(C) Springer-Verlag Berlin Heidelberg 2015

\begin{abstract}
Purpose Adenoma is the main parathyroid disorder leading to primary hyperparathyroidism (PHP). Minimally invasive parathyroidectomy (MIP) is recognized as a valid procedure for adenoma-related PHP. It requires precise preoperative localization combining Tc-99m-MIBI (methoxy-isobutylisonitrile) scintigraphy and single-photon emission computed tomography (SPECT) with x-ray computed tomography (CT) and intraoperative confirmation of successful excision by change in intact parathormone (iPTH) levels. The study aim was to assess the surgery success in relation to these two parameters. Methods All patients operated on for PHP from 2005 to mid2014 at our institution were retrospectively reviewed. MIP was performed in case of precise preoperative adenoma localization on scintigraphy, absence of past cervical surgery, and absence of concomitant thyroid resection necessity. In these patients, iPTH levels were monitored intraoperatively. Confirmation criteria for iPTH values were a return to normal level or a decrease $>50 \%$ of basal iPTH level.

Results There were 197 PHP operations during the study period: $118 \mathrm{MIP}$ and 79 bilateral neck explorations (BNEs). The MIP success rate was $95 \%(112 / 118)$ with a preoperative
\end{abstract}

This paper has been presented in parts at the 101st Annual Congress of the Swiss Surgical Society, May 21-23, 2014, Bern, Switzerland.

Maurice Matter

maurice.matter@chuv.ch

1 Department of Visceral Surgery, University Hospital CHUV, Rue du Bugnon 46, 1011 Lausanne, Switzerland

2 Department of Endocrinology, University Hospital CHUV, Lausanne, Switzerland

3 Department of Nuclear Medicine, University Hospital CHUV, Lausanne, Switzerland
MIBI scan $\pm \mathrm{CT}$ accurate in $94 \%(111 / 118)$ of the patients and with correct iPTH in $90 \%(106 / 118)$ of the cases. Among the $12 \mathrm{iPTH}$ levels that did not meet the confirmation criteria, 10 returned to normal range by postoperative day 2 . Treatment failure appeared in three patients (one BNE, two MIPs). Conclusions Tc-99m-MIBI dual-phase scintigraphy with SPECT/CT is the key examination for functional and morphological parathyroid adenoma localization. If preoperative scintigraphy is obvious and intraoperative assessment is clear, one could possibly safely omit iPTH, as it may lead to unnecessary BNE in primary PHP.

Keywords Parathyroidectomy · Scintigraphy $\cdot$ Intraoperative parathyroid level $\cdot$ Minimally invasive surgery

\section{Introduction}

Primary hyperparathyroidism (PHP) is the main disorder of the parathyroid glands $[1,2]$. Adenoma represents the most frequent cause of PHP (between 70 and $85 \%$ [3]. The development of surgical techniques of parathyroid gland excision over the last two decades has allowed performing a focused surgery, thus avoiding systematic bilateral neck exploration (BNE) and its concomitant morbidity, mainly transient hypocalcemia and scar-related problems [4-6]. In parallel, imaging technologies have also progressed. The development of hybrid cameras (PET/CT, single-photon emission computed tomography with $\mathrm{x}$-ray computed tomography (SPECT/CT)) has improved the anatomical localization of functionally detected abnormalities. The adjunct of delayed SPECT acquisition during the Tc-99mMIBI (methoxy-isobutyl-isonitrile) dual-phase scintigraphy has improved both the resolution and the sensitivity of the study to detect and localize adenomas when compared 
to planar imaging $[7,8]$. Hybrid cameras can precisely and specifically localize the adenoma by avoiding false-positive results of Tc-99m-MIBI SPECT [9-11].

The developments of minimally invasive surgery have included the mini-cervicotomy (midline or just above the localized adenoma) and the cervicoscopy. These minimally invasive techniques have been demonstrated as valid and efficient procedures [3, 12-16]. They presuppose a precise preoperative localization of the adenoma and an intraoperative confirmation of an adequate resection. Preoperative imaging investigations with dual-phase Tc-99m-MIBI scintigraphy with SPECT/CT and ultrasound (US) can give important anatomical details on an adenoma location. Intraoperative confirmation of adenoma resection includes frozen section and intraoperative changes of intact parathormone (iPTH) plasma levels 10 and 15 min after resection $[17,18]$.

The aim of this study was to evaluate the impact and quality of MIBI scans and the impact of iPTH levels on the success of minimally invasive parathyroidectomy (MIP). MIP results were also compared to a BNE group in order to have a control group.

\section{Material and methods}

At our institution, dual-phase Tc-99m-MIBI scintigraphy with pinhole acquisitions, SPECT, and thyroid scintigraphy are routinely performed since the late 1990s. MIP was introduced in 2005, and since 2008, we changed from SPECT to SPECT/ CT.

All consecutive patients operated on for PHP at our tertiary referral center from January 2005 to June 2014 were retrospectively reviewed. All the operations were performed by the same dedicated endocrine surgeon.

Data collection included the following: preoperative characteristics (age, gender, serum calcium level, serum parathormone level, body mass index, American Society of Anesthesiologists score, comorbidities), imaging results (US, Tc-99mMIBI scintigraphy), intraoperative findings (conversion rate, adenoma size and weight, operating time), intraoperative iPTH levels, and postoperative outcomes (serum calcium level, complications, length of hospital stay, and follow-up). US and Tc-99m-MIBI SPECT \pm CT scintigraphy were performed in all patients. US was primarily used not only to identify the adenoma but also to detect any suspicious thyroid nodule that could alter the imaging and surgical strategy. A preoperative and postoperative otolaryngologic examination was performed on every operated patient. Postoperative complications were rated according to the Dindo-Clavien classification [19]. Hypocalcemia was defined as a serum calcium level $<2.15 \mathrm{mmol} / \mathrm{l}$ regardless of the symptoms. The definitive diagnosis was based on the histopathological results.
Operations consisted of MIP or BNE. MIP included focused mini-cervicotomy (between the sternocleidomastoid and the infrahyoid muscles) and cervicoscopy according to Henry's technique [20]. Mini-cervicotomy was performed with a $2-\mathrm{cm}$ incision, whereas Henry's technique comprised one 11-mm trocar for the camera and two 3-mm working trocars. Criteria to undertake MIP were as follows: preoperative detection of an adenoma and precise localization of the adenoma with MIBISPECT \pm CT. Criteria to undertake BNE were as follows: absence of definitive adenoma localization, previous cervical surgery, or need of concomitant thyroid surgery.

Peripheral venous samplings for intraoperative iPTH levels were performed just before neck mobilization and surgical skin disinfection (T0), at $10 \mathrm{~min}$ (T10), and $15 \mathrm{~min}$ (T15) after excision of the adenoma. Criteria for iPTH were based on the Rome criteria [21]. Return to normal range or decrease of more than $50 \%$ at $\mathrm{T} 10 / \mathrm{T} 15$ compared to initial $\mathrm{T} 0$ values was used as safety criteria. Testing was performed in the endocrinology unit laboratory with a 30-min mean delay for the results.

Because of the high selection of the patients in the MIP group (highly selective MIBI-SPECT-CT), some patients who did not fulfill the Rome criteria were followed during $24 \mathrm{~h}$ with calcium and standard iPTH measures on postoperative day 1 .

In the MIP group, conversion was defined as the need of undertaking a BNE with standard cervicotomy. Success rate of MIP was defined as no need of conversion to BNE. Treatment failure for MIP and BNE was defined as long-term (more than 6 months) persistence of hypercalcemia and high level of parathyroid hormone after the operation (no resolution of PHP postoperatively) [22].

Intraoperative levels of parathormone measured the 1-84 iPTH using a two-step sandwich solid-phase enzyme immunoassay (ARCHITECT ${ }^{\odot}$ iPTH assay, Abbott Diagnostic Division, USA). The 1-84 iPTH half-life ranges between 4 and 5 min [23-28]. The iPTH is independent of the glomerular filtration rate and represents the biologically active hormone part [29]. Early postoperative iPTH and calcium levels were measured during hospitalization. Laboratory follow-up tests were not performed routinely but just if indicated.

Statistical analyses were performed using GraphPad Prism $^{\odot} 5$ for Mac OS X. A $p$ value $<0.05$ was considered statistically significant. Mann-Whitney $U$ test or Fisher's exact test was used to compare the two groups according to the distribution and variable types. Sensitivity, specificity, positive predictive value, and negative predictive value of Tc99m-MIBI scintigraphy for adenoma were also calculated. Positive predictive value was defined as the number of true positives divided by the number of true positives plus the number of false positives. Negative predictive value was calculated as the number of true negatives divided by the number of true negatives plus the number of false negatives. 


\section{Results}

Patients' characteristics, surgical results, and postoperative data of both groups (MIP and BNE) are summarized in Table 1. Sixty-eight patients had an adenoma in the BNE group and 111 in the MIP group, while seven and five had parathyroid hyperplasia, respectively. There was no difference in terms of demographics between the two groups except for the body mass index. All frozen section results corresponded to final pathological reports. In the 179 MIBI scintigraphies performed in both groups (MIP and BNE) for adenomas, 126 (104 in the MIP group and 22 in the BNE group) were positive (correct localization) and 53 (7 and 46, respectively) were negative. Between the negative and positive MIBI scintigraphies, there were no differences in terms of gland size, gland weight, intraoperative iPTH levels, and calcium levels.

Between the two groups, operating time (including only BNE without thyroid resection) was shorter for MIP $(p<0.01)$, length of hospital stay was 1 day shorter for MIP $(p<0.01)$, and median follow-up was longer for $\mathrm{BNE}$ $(p<0.01)$. There was no difference in the other postoperative outcomes. Median (IQR) calcium levels on postoperative day 1 were $2.16 \mathrm{mmol} / \mathrm{l}(2.07-2.31)$ for the MIP group and
$2.17 \mathrm{mmol} / \mathrm{l}(2.01-2.29)$ for the BNE group. Postoperatively, iPTH levels were not routinely measured. There were four patients with multiple endocrine neoplasia (MEN) 2A in the BNE group.

Double adenoma was observed in two patients (one with initial BNE and one with MIP necessitating a conversion to $\mathrm{BNE}$ ), whereas triple adenoma was present in one BNE patient. Regarding the case of triple adenoma, three glands were found to be adenomatous, and the fourth one was judged microscopically normal by the pathologist. No specific genetic mutations were found. Carcinoma was found in one BNE patient. Normal parathyroid tissue was found in one BNE patient and one MIP patient (Table 1).

Treatment failure, i.e., no resolution of PHP postoperatively, occurred in three patients (two in the MIP group and one in the initial BNE group). One patient with a mediastinal adenoma in the thymus and a positive selective venous sampling was successfully operated on by thoracoscopy, one patient had a new imaging with MIBI-SPECT/CT and was subsequently successfully reoperated on (right inferior adenoma), and one patient is still being worked up after a negative cervicomediastinal MRI (waiting for a selective venous
Table 1 Preoperative results, intraoperative findings, and postoperative data for bilateral neck exploration (BNE) patients and minimally invasive parathyroidectomy (MIP) patients

\begin{tabular}{|c|c|c|c|}
\hline & BNE $N=79$ & $\operatorname{MIP} N=118$ & $p$ value \\
\hline Age (years) & $65(25-88)$ & $60(20-89)$ & 0.12 \\
\hline Body mass index $\left(\mathrm{kg} / \mathrm{m}^{2}\right)$ & $27.7(18.2-50.4)$ & $24.2(16.8-43.6)$ & $<0.01$ \\
\hline ASA score I/II/III & $2 / 65 / 12$ & $8 / 93 / 17$ & 0.41 \\
\hline Preoperative $\mathrm{Ca}^{++}(N 2.15-2.55 \mathrm{mmol} / \mathrm{l})$ & $2.76(2.30-4.30)$ & $2.77(2.35-4.06)$ & 0.31 \\
\hline Preoperative iPTH (N 10-70 ng/l) & $115(9-2006)$ & $126(11-745)$ & 0.71 \\
\hline MIBI scintigraphy performed & 73 & 118 & - \\
\hline \multicolumn{4}{|l|}{ Reasons for BNE } \\
\hline 1. No preoperative localization & 32 & & \\
\hline 2. Thyroid surgery needed & 42 & & \\
\hline 3. Past cervical surgery & 5 & & \\
\hline Adenoma & 68 & 111 & 0.08 \\
\hline Hyperplasia & 7 & 5 & 0.23 \\
\hline Other pathology results ${ }^{\mathrm{a}}$ & 4 & 2 & 0.22 \\
\hline Operating time (min) & $96(36-212)^{\mathrm{b}}$ & $59(20-200)$ & $<0.01$ \\
\hline Gland size $(\mathrm{cm})$ & $1.8(0.2-5)$ & $1.6(0.7-6)$ & 0.86 \\
\hline Gland weight (mg) & $699(10-13,200)$ & $900(0.59-6,300)$ & 0.15 \\
\hline Postoperative hypocalcemia & 29 & 31 & 0.15 \\
\hline Transitory/permanent laryngeal nerve palsy & $7 / 2$ & $3 / 1$ & 0.09 \\
\hline Treatment failure & 1 & 2 & 0.81 \\
\hline Length of hospital stay (days) & $2(1-28)$ & $1(1-20)$ & $<0.01$ \\
\hline Length of follow-up (months) & $66.8(6.3-121.2)$ & $39.6(6.2-118.1)$ & $<0.01$ \\
\hline
\end{tabular}

Number of cases and medians with range

${ }^{a}$ Double adenoma (2), triple adenoma (1), carcinoma (1), or normal tissue (2)

${ }^{\mathrm{b}}$ Only BNEs without concomitant thyroid resection were taken into account $(n=37)$ 
sampling). The median follow-up was 66.8 months for BNE and 39.6 months for MIP.

The sensitivity of Tc-99m-MIBI scintigraphy for adenomas (179 adenomas in the BNE and MIP groups) was in our cohort: $126 /(126+53)=70 \%$. The number of true positives was 126 (adenomas in both groups with correct preoperative imaging), and the number of false negatives was 53 (adenomas in both groups with incorrect preoperative imaging). The specificity was $44 \%(8 /(8+10))$, where 8 represents the number of true negatives (i.e., the number of non-adenoma resections with negative preoperative imaging) and 10 the number of false positives (i.e., the number of non-adenoma resections with positive imaging for adenoma). The positive predictive value was $93 \%(126 /(126+10))$ and the negative predictive value $16 \%(8 /(8+53))$.

Table 2 shows the imaging results and outcomes of MIP (divided into cervicoscopy and mini-cervicotomy). Thirty-one patients underwent cervicoscopy and 87 mini-cervicotomy. One treatment failure occurred after cerviscopy and one after mini-cervicotomy. There was no difference in terms of age, body mass index, American Society of Anesthesiologists score, preoperative calcium and iPTH levels, operating time, pathology results, length of stay, conversion rate, and complication rate between the cervicoscopy and mini-cervicotomy patients. Seventy-eight of 118 (66\%) patients had a correct preoperative US localization and could have been selected for MIP only based on US. In the MIP group, Tc-99m-MIBI scintigraphy \pm CT correctly localized the adenoma in $94 \%$ of the cases (111/118). Six patients had a conversion to BNE. Intraoperative iPTH levels fulfilled Rome criteria (return to normal range at $\mathrm{T} 15$ or decay of more than $50 \%$ at $\mathrm{T} 15)$ in $90 \%$ of the cases (106/118). In the MIP group, median operating time was shorter for the 96 patients who had a preoperative SPECT/CT (54 min, range 20-200 $\mathrm{min}$ ) compared to the 22 patients who only had a SPECT (74.5 min, range 43$172 \mathrm{~min}, p=0.0005$ ). If one compares the median operating time of the five first years of the study period (2005 to 2009) to the five last years (2010 to 2014), a statistically significant difference is found ( 72 vs. $53 \mathrm{~min}, p=0.001$ ). The MIP learning curve is represented in Fig. 1.

The reasons for the six conversions to BNE were as follows: absence of finding of the lesion intraoperatively (3), double adenoma (1), necessity of thyroid resection due to strong adhesion of the parathyroid adenoma (1, right thyroid lobectomy), and suspicion of multiglandular disease (1). In the six converted patients, MIBI imaging was inaccurate in three cases. BNE leads to correct resection in four patients and to treatment failure in the two other patients.
Table 2 Imaging results and outcomes of the minimally invasive parathyroidectomy (MIP) patient group

\begin{tabular}{lll}
\hline & \multicolumn{2}{l}{ MIP N=118 } \\
\cline { 2 - 3 } & Cervicoscopy ${ }^{\mathrm{a}} N=31$ & Mini-cervicotomy $N=87$ \\
\hline Preoperative US & $31(100 \%)$ & $87(100 \%)$ \\
True positives US localization & $21(68 \%)$ & $57(66 \%)$ \\
Preoperative MIBI-SPECT only (without CT) & $0(0 \%)$ & $22(25 \%)$ \\
Preoperative MIBI-SPECT/CT & $31(100 \%)$ & $65(75 \%)$ \\
True positives MIBI-SPECT localization & $0(0 \%)$ & $20(91 \%)$ \\
True positives MIBI-SPECT/CT localization & $29(94 \%)$ & $62(95 \%)$ \\
Conversion to bilateral neck exploration (BNE) & $1(3 \%)$ & $5(6 \%)$ \\
Intraoperative iPTH & & \\
1. Decay (10-15 min) $>50 \%$ & $26(84 \%)$ & $77(89 \%)$ \\
2. Return to normal range & $25(81 \%)$ & $74(85 \%)$ \\
3. Satisfying criteria 1 or 2 & $28(90 \%)$ & $78(90 \%)$ \\
4. Not satisfying criteria 1 or 2 & $3(10 \%)$ & $9(10 \%)$ \\
5. Among 4 return to normal range on days $1-2$ & $2(6 \%)$ & $8(9 \%)$ \\
Postoperative hypocalcemia & $6(19 \%)$ & $25(29 \%)$ \\
Complications (Dindo-Clavien) & & $76(87 \%)$ \\
No & $27(87 \%)$ & $11(13 \%)$ \\
Minor (I-II) & & $0(0 \%)$ \\
Major (III-V) & $4(13 \%)$ & \\
\hline
\end{tabular}

a According to Henry's technique

${ }^{b}$ Number of patients fulfilling each item. 3 and 4 give the total number of patients

${ }^{\mathrm{c}}$ Laryngeal nerve palsy and others. Hypocalcemia was not considered as a complication 


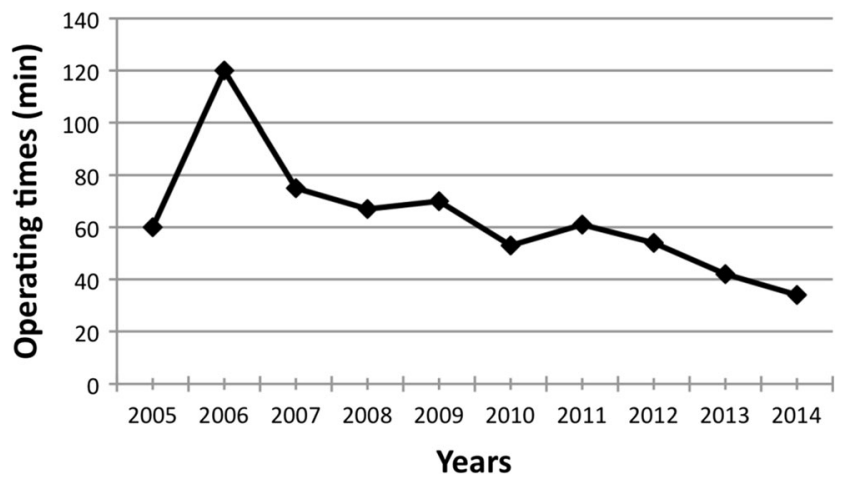

Fig. 1 Median operating times of minimally invasive parathyroidectomies throughout the years (learning curve)

The success rate of MIP was $95 \%$ (112/118) with a preoperative MIBI scan accurate in $94 \%(111 / 118)$ of the MIP patients and with correct iPTH in $90 \%$ (106/118) of the cases. Among the 12 cases not satisfying the criteria, 10 patients had normal iPTH values on day 1 or 2 with no treatment failure during the follow-up.

Seven patients in the MIP group had ultimately a preoperative localization that was not correct: four had to be converted to BNE (one suspicion of multiglandular disease and three adenomas not found intraoperatively) and three had a successful MIP as they were on the same side (inferior on scintigraphy and superior during surgery, Table 3).

\section{Discussion}

This study showed that a high success rate of MIP $(95 \%)$ was associated with a $94 \%$ accurate detection rate of the preoperative MIBI scan and with a $90 \%$ rate of correct intraoperative iPTH measures in our cohort. It therefore demonstrates that MIBI scan imaging and intraoperative iPTH measures importantly correlate with the success of MIP.

PHP prevalence is $0.86 \%$ in the USA and $0.73 \%$ in Europe [2, 30-32]. In approximately $80 \%$ of PHP patients, the etiology is an adenoma, followed by parathyroid hyperplasia (multiglandular disease) and parathyroid cancer [3]. In the present study including 197 PHP patients, 179 had an adenoma, two a double adenoma, one a triple adenoma, one a carcinoma, 12 hyperplasia, and two normal tissue, well in line with other published series [2,31,33]. This corresponds to an epidemiological change. Indeed, the adenoma rate has been increasing for the last decades whereas multiglandular disease has become less frequent. The latter still remains a diagnostic challenge.

In this series, $3 \%$ of patients (4/118) who underwent MIP had a postoperative recurrent laryngeal nerve palsy. Among these four patients, three had a transient palsy, and only one a

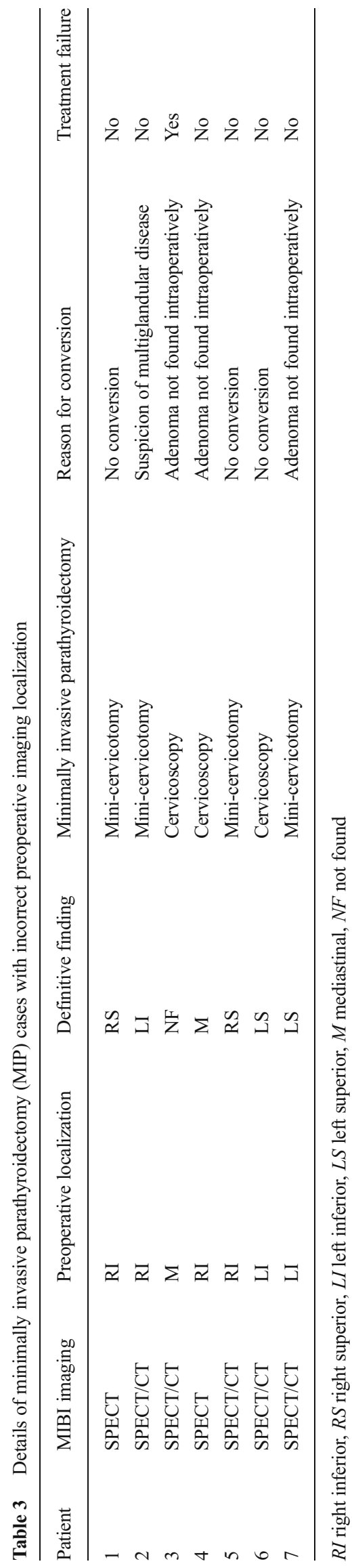


permanent palsy. This is in the range of what is usually found in the literature after MIP $[15,20]$. Of note, these figures were found due to a strict policy of preoperative and postoperative otolaryngologic controls performed in every patient.

In the MIP group, MIBI-SPECT \pm CT localization of the adenoma was correct and accurate in $94 \%$ of the patients. According to our data, MIBI-SPECT \pm CT sensitivity for adenoma was $70 \%$ which is similar to other study findings [9-11, 13]. It is important to mention that the adenoma prevalence does not influence the sensitivity but has an impact on the predictive values. This should be kept in mind when interpreting the results of a diagnostic test. Based on our results MIBI scintigraphy represents an accurate preoperative imaging to localize a parathyroid adenoma. It is therefore an essential tool for a successful minimally invasive surgery. In this setting and according to our results, it is important to combine MIBI scintigraphy with CT (SPECT/CT) in order to have a 3D anatomical view specifying the adenoma localization and its relation to the adjacent organs. This advantage of SPECT/CT over SPECT resulted in a shorter operating time (decrease of the median operating time of $20.5 \mathrm{~min}, p=0.0005$ ). A MIBISPECT with only antero-posterior views can sometimes be misleading without CT (Fig. 2). In our group, SPECT alone was able to precisely locate the adenoma in 20 out of 22 patients. This highlights that the number of true positive adenoma detection mainly depends on the MIBI captation.

The plasma levels of iPTH regained normal values or decreased more than $50 \%$ at T10/15 in $90 \%$ of the cases. These measurements are a good help in MIP to confirm the successful excision of the adenoma. Despite the above-mentioned guidelines and because we trust the highly sensitive SPECT/ CT, it was decided to stop surgery in 12 patients with unmet criteria. Ten out of these 12 patients who did not satisfy Rome criteria reached normal iPTH levels 1 or 2 days after surgery and stayed like that during the follow-up. One hypothesis is that in these ten patients, the ARCHITECT ${ }^{\odot}$ method measured other forms of iPTH (e.g., 2-84, 3-84) or "almost intact" forms of PTH that have longer half-lives. Of note, the crucial element that can alter the iPTH measures is preanalytical. Enzymatic degradation of iPTH mainly depends on conservation temperature and time between sampling and analysis. Important hemolysis $(>5 \mathrm{~g} / \mathrm{l})$, hypertriglyceridemia $(>50 \mathrm{~g} / \mathrm{l})$, and hyperbilirubinemia $(>200 \mathrm{mg} / \mathrm{l})$ can also alter iPTH measurements. No such factors were found in these 12 patients, and with a median follow-up of 36 months (range 6123 ), only two had a definitive treatment failure. By trusting the preoperative localization and the intraoperative assessment, unnecessary BNE was avoided in 10 out of 12 patients. The iPTH measurement is mainly performed to detect the presence of rarely encountered double adenoma or multiglandular disease [34-36]. In the 197 PHP patients, we observed only two cases of double adenoma, one case of triple adenoma, and 12 cases of multiglandular disease.

Different criteria to assess the intraoperative $\mathrm{iPTH}$ changes have been published (Rome, Vienna, Miami, Halle, etc.) [17, $21,37-42]$. None can perfectly exclude multiglandular disease
Fig. 2 Two cases of MIBISPECT showing a left lower parathyroid adenoma (left side imaging pictures). When coupled with a CT-scan (SPECT/CT on the right side), the upper lesion still appears to be in the left lower parathyroid gland while the other lies in the mediastinum on the aorta. This illustrates the importance to couple MIBISPECT with a CT scan for precise preoperative localization of an adenoma

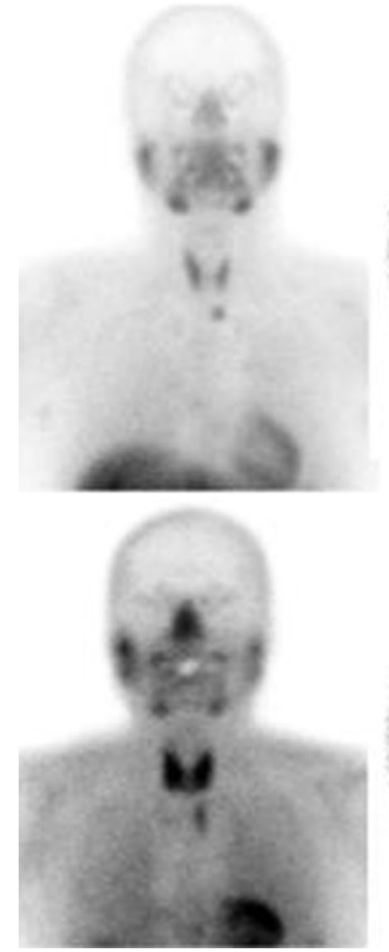

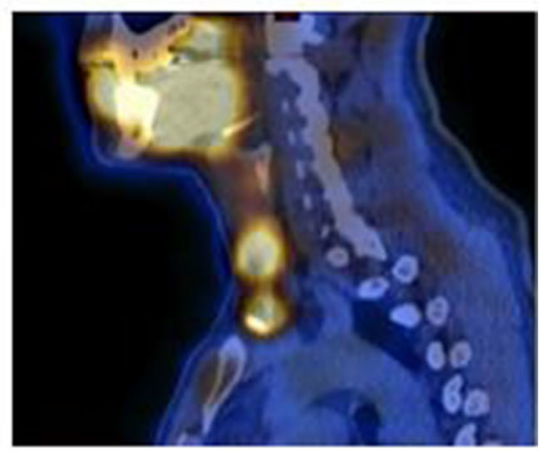

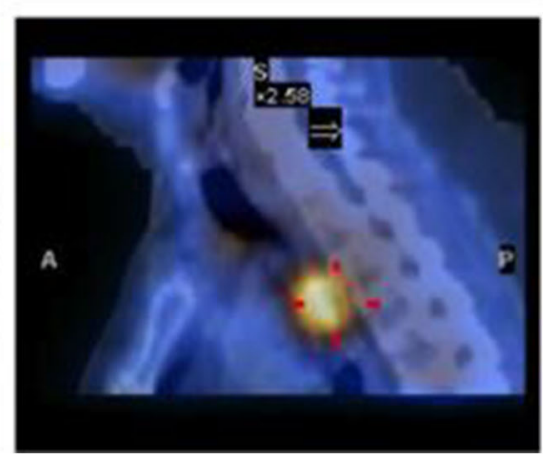


or double adenoma, while safely confirm a single adenoma [38]. If the criteria are more drastic, then the risk of performing unnecessary BNE is higher. On the contrary, if the criteria are satisfied too easily, certain adenomas can be missed [43]. The complete abandon of intraoperative iPTH levels, suggested by our results, was already proposed by Twigt et al. [44]. They concluded that even though intraoperative iPTH level can be helpful in some cases, it should not be routinely performed as the iPTH levels would have potentially changed the outcomes of only $1.7 \%$ of their patients (2/119) [44].

US results for adenoma localization were correct in $66 \%(78 / 118)$ of the MIP cases. US is not as precise as MIBI-SPECT/CT and has limitations to localize lower or posterior glands. However, it has a good sensitivity to detect thyroid nodules and represents the technique of choice for a first cervical workup [9, 45]. Simultaneous thyroid disease with PHP is indeed not that uncommon. The need of concomitant thyroid resection in case of PHP was observed in $22 \%(43 / 197)$ in our series and is around $30 \%$ in the literature [46, 47]. In case of clear positive US precisely localizing an adenoma, a risk-benefit evaluation of the need of MIBI-SPECT/CT should be performed. MIBI-SPECT/CT indeed induces radiation exposure and has an elevated cost.

In case of treatment failure, several options can be envisaged like selective venous sampling or novel imaging (SPECT/CT, MRI). In our institution, we usually first repeat the SPECT/CT. If it is not conclusive, an MRI or a selective venous sampling is performed.

A limitation of this study was its retrospective design. Some data were not available and not found in the patient charts. Moreover, as mentioned in the methods, no routine follow-up tests (iPTH and calcium levels) were performed if there was no indication.

In conclusion, Tc-99m-MIBI dual-phase scintigraphy with SPECT/CT accurately localizes a parathyroid adenoma allowing to perform MIP with a high success rate. Usefulness of intraoperative iPTH levels should be balanced by the fact that BNE could be an unnecessary consequence of unmet criteria. If the preoperative imaging is precise and the intraoperative finding is clear, unmet intraoperative iPTH criteria do not induce an obligatory BNE. Based on our findings, a waitand-see policy with $\mathrm{PTH}$ measure on postoperative day 1 could be a valid option.

Authors' contributions GRJ: study conception and design, acquisition of data, analysis and interpretation of data, drafting of the manuscript, and acceptance of the final version.

ND: study conception and design, analysis and interpretation of data, critical revision of the manuscript, and acceptance of the final version.

LP: analysis and interpretation of data, critical revision of the manuscript, and acceptance of the final version.

$\mathrm{AB}$ : analysis and interpretation of data, critical revision of the manuscript, and acceptance of the final version.
MM: study conception and design, acquisition of data, analysis and interpretation of data, critical revision of the manuscript, and acceptance of the final version.

Compliance with ethical standards - This study received no particular funding.

- This retrospective study based on chart review was in accordance with the ethical standards of the institutional research committee and with the 1964 Helsinki declaration and its later amendments or comparable ethical standards.

- Informed consent was obtained from all individual participants included in the study.

\section{Sources of financial support None.}

Conflicts of interest Gaëtan-Romain Joliat, Nicolas Demartines, Luc Portmann, Ariane Boubaker, and Maurice Matter have no conflicts of interest to disclose.

\section{References}

1. Clarke BL (2013) Epidemiology of primary hyperparathyroidism. J Clin Densitom 16:8-13

2. Press DM, Siperstein AE, Berber E, Shin JJ, Metzger R, Monteiro $R$ et al (2013) The prevalence of undiagnosed and unrecognized primary hyperparathyroidism: a population-based analysis from the electronic medical record. Surgery 154:1232-1237

3. Hessman O, Westerdahl J, Al-Suliman N, Christiansen P, Hellman P, Bergenfelz A (2010) Randomized clinical trial comparing open with video-assisted minimally invasive parathyroid surgery for primary hyperparathyroidism. Br J Surg 97:177-184

4. Rio PD, Vicente D, Maestroni U, Totaro A, Pattacini GMC, Avital I et al (2013) A comparison of minimally invasive video-assisted parathyroidectomy and traditional parathyroidectomy for parathyroid adenoma. J Cancer 4:458-463

5. Tibblin S, Bondeson AG, Ljungberg O (1982) Unilateral parathyroidectomy in hyperparathyroidism due to single adenoma. Ann Surg 195:245-252

6. Bergenfelz A, Lindblom P, Tibblin S, Westerdahl J (2002) Unilateral versus bilateral neck exploration for primary hyperparathyroidism: a prospective randomized controlled trial. Ann Surg 236:543-551

7. Staudenherz A, Abela C, Niederle B, Steiner E, Helbich T, Puig S et al (1997) Comparison and histopathological correlation of three parathyroid imaging methods in a population with a high prevalence of concomitant thyroid diseases. Eur J Nucl Med 24:143-149

8. Sfakianakis GN, Irvin GL, Foss J, Mallin W, Georgiou M, Deriso GT et al (1996) Efficient parathyroidectomy guided by SPECTMIBI and hormonal measurements. J Nucl Med 37:798-804

9. Akbaba G, Berker D, Isik S, Aydin Y, Ciliz D, Peksoy I et al (2012) A comparative study of pre-operative imaging methods in patients with primary hyperparathyroidism: ultrasonography, $99 \mathrm{mTc}$ sestamibi, single photon emission computed tomography, and magnetic resonance imaging. J Endocrinol Investig 35(4):359-364

10. Tokmak H, Demirkol MO, Alagöl F, Tezelman S, Terzioglu T (2014) Clinical impact of SPECT-CT in the diagnosis and surgical management of hyper-parathyroidism. Int J Clin Exp Med 7(4): 1028-1034

11. Swanson TW, Chan SK, Jones SJ, Bugis S, Irvine R, Belzberg A et al (2010) Determinants of Tc-99m sestamibi SPECT scan sensitivity in primary hyperparathyroidism. Am J Surg 199:614-620 
12. Slepavicius A, Beisa V, Janusonis V, Strupas K (2008) Focused versus conventional parathyroidectomy for primary hyperparathyroidism: a prospective, randomized, blinded trial. Langenbecks Arch Surg 393:659-666

13. Westerdahl J, Bergenfelz A (2007) Unilateral versus bilateral neck exploration for primary hyperparathyroidism: five-year follow-up of a randomized controlled trial. Ann Surg 246:976-980

14. Bergenfelz A, Kanngiesser V, Zielke A, Nies C, Rothmund M (2005) Conventional bilateral cervical exploration versus open minimally invasive parathyroidectomy under local anaesthesia for primary hyperparathyroidism. Br J Surg 92:190-197

15. Barczyński M, Cichoń S, Konturek A, Cichoń W (2006) Minimally invasive video-assisted parathyroidectomy versus open minimally invasive parathyroidectomy for a solitary parathyroid adenoma: a prospective, randomized, blinded trial. World J Surg 30:721-731

16. Miccoli P, Bendinelli C, Berti P, Vignali E, Pinchera A, Marcocci C (1999) Video-assisted versus conventional parathyroidectomy in primary hyperparathyroidism: a prospective randomized study. Surgery 126:1117-1121

17. Irvin GL, Dembrow VD, Prudhomme DL (1993) Clinical usefulness of an intraoperative "quick parathyroid hormone" assay. Surgery 114:1019-1022

18. Bergenfelz A, Nordén NE, Ahrén B (1991) Intraoperative fall in plasma levels of intact parathyroid hormone after removal of one enlarged parathyroid gland in hyperparathyroid patients. Eur J Surg 157:109-112

19. Dindo D, Demartines N, Clavien P-A (2004) Classification of surgical complications: a new proposal with evaluation in a cohort of 6336 patients and results of a survey. Ann Surg 240:205-213

20. Henry J-F, Sebag F, Tamagnini P, Forman C, Silaghi H (2004) Endoscopic parathyroid surgery: results of 365 consecutive procedures. World J Surg 28:1219-1223

21. Lombardi CP, Raffaelli M, Traini E, Di Stasio E, Carrozza C, De Crea $\mathrm{C}$ et al (2008) Intraoperative PTH monitoring during parathyroidectomy: the need for stricter criteria to detect multiglandular disease. Langenbecks Arch Surg 393:639-645

22. Lee S, Ryu H, Morris LF, Grubbs EG, Lee JE, Harun N et al (2014) Operative failure in minimally invasive parathyroidectomy utilizing an intraoperative parathyroid hormone assay. Ann Surg Oncol 21: 1878-1883

23. Stalberg P, Sidhu S, Sywak M, Robinson B, Wilkinson M, Delbridge L (2006) Intraoperative parathyroid hormone measurement during minimally invasive parathyroidectomy: does it "valueadd" to decision-making? J Am Coll Surg 203:1-6

24. Carter AB, Howanitz PJ (2003) Intraoperative testing for parathyroid hormone: a comprehensive review of the use of the assay and the relevant literature. Arch Pathol Lab Med 127:1424-1442

25. Bieglmayer C, Prager G, Niederle B (2002) Kinetic analyses of parathyroid hormone clearance as measured by three rapid immunoassays during parathyroidectomy. Clin Chem 48:1731-1738

26. Ito F, Sippel R, Lederman J, Chen H (2007) The utility of intraoperative bilateral internal jugular venous sampling with rapid parathyroid hormone testing. Ann Surg 245:959-963

27. Emmolo I, Corso HD, Borretta G, Visconti G, Piovesan A, Cesario $F$ et al (2005) Unexpected results using rapid intraoperative parathyroid hormone monitoring during parathyroidectomy for primary hyperparathyroidism. World J Surg 29:785-788

28. Sharma J, Milas M, Berber E, Mazzaglia P, Siperstein A, Weber CJ (2008) Value of intraoperative parathyroid hormone monitoring. Ann Surg Oncol 15:493-498

29. Goltzman D, Hendy G (2001) Parathyroid hormone. Principles and practice of endocrinology and metabolism, 3rd edn. Lippincott Williams \& Wilkins, Philadelphia, pp 497-512

30. Abood A, Vestergaard P (2013) Increasing incidence of primary hyperparathyroidism in Denmark. Dan Med J 60:A4567
31. Siilin H, Lundgren E, Mallmin H, Mellström D, Ohlsson C, Karlsson M et al (2011) Prevalence of primary hyperparathyroidism and impact on bone mineral density in elderly men: MrOs Sweden. World J Surg 35: 1266-1272

32. Richert L, Trombetti A, Herrmann FR, Triponez F, Meier C, Robert $\mathrm{JH}$ et al (2009) Age and gender distribution of primary hyperparathyroidism and incidence of surgical treatment in a European country with a particularly high life expectancy. Swiss Med Wkly 139: 400-404

33. Twigt BA, Vollebregt AM, van Dalen T, Smits AB, Consten ECJ, van Vroonhoven TJMV et al (2011) Shifting incidence of solitary adenomas in the era of minimally invasive parathyroidectomy. A multi-institutional study. Ann Surg Oncol 18:1041-1046

34. Clerici T, Brandle M, Lange J, Doherty GM, Gauger PG (2004) Impact of intraoperative parathyroid hormone monitoring on the prediction of multiglandular parathyroid disease. World J Surg 28: 187-192

35. Abboud B, Sleilaty G, Helou E, Mansour E, Tohme C, Noun R et al (2005) Existence and anatomic distribution of double parathyroid adenoma. Laryngoscope 115:1128-1131

36. Milas M, Wagner K, Easley KA, Siperstein A, Weber CJ (2003) Double adenomas revisited: nonuniform distribution favors enlarged superior parathyroids (fourth pouch disease). Surgery 134: 995-1000

37. Papier A, Kenig J, Barczyński M (2014) Evaluation of different intraoperative iPTH assay criteria in monitoring of minimally invasive parathyroidectomy for primary hyperparathyroidism. Przegla d Lek 71:14-18

38. Barczynski M, Konturek A, Hubalewska-Dydejczyk A, Cichon S, Nowak W (2009) Evaluation of Halle, Miami, Rome, and Vienna intraoperative $\mathrm{PTH}$ assay criteria in guiding minimally invasive parathyroidectomy. Langenbecks Arch Surg 394:843-849

39. Carneiro DM, Irvin GL (2002) New point-of-care intraoperative parathyroid hormone assay for intraoperative guidance in parathyroidectomy. World J Surg 26:1074-1077

40. Riss P, Kaczirek K, Heinz G, Bieglmayer C, Niederle B (2007) A "defined baseline" in PTH monitoring increases surgical success in patients with multiple gland disease. Surgery 142:398-404

41. Irvin GL, Solorzano CC, Carneiro DM (2004) Quick intraoperative parathyroid hormone assay: surgical adjunct to allow limited parathyroidectomy, improve success rate, and predict outcome. World J Surg 28:1287-1292

42. Carneiro DM, Solorzano CC, Nader MC, Ramirez M, Irvin GL (2003) Comparison of intraoperative iPTH assay (QPTH) criteria in guiding parathyroidectomy: which criterion is the most accurate? Surgery 134:973-979

43. Mihai R, Palazzo FF, Gleeson FV, Sadler GP (2007) Minimally invasive parathyroidectomy without intraoperative parathyroid hormone monitoring in patients with primary hyperparathyroidism. $\mathrm{Br}$ J Surg 94:42-47

44. Twigt BA, van Dalen T, Vollebregt AM, Kortlandt W, Vriens MR, Borel Rinkes IHM (2012) The additional value of intraoperative parathyroid hormone assessment is marginal in patients with nonfamilial primary hyperparathyroidism: a prospective cohort study. Am J Surg 204:1-6

45. Schenk WG, Hanks JB, Smith PW (2013) Surgeon-performed ultrasound for primary hyperparathyroidism. Am Surg 79:681-685

46. Ryan S, Courtney D, Timon C (2014) Co-existent thyroid disease in patients treated for primary hyperparathyroidism: implications for clinical management. Eur Arch Oto-Rhino-Laryngol 272:419-423

47. Gómez-Ramírez J, Sancho-Insenser JJ, Pereira JA, Jimeno J, Munné A, Sitges-Serra A (2010) Impact of thyroid nodular disease on $99 \mathrm{mTc}$-sestamibi scintigraphy in patients with primary hyperparathyroidism. Langenbecks Arch Surg 395:929-933 